giform encephalopathies and that a modified form of PrP is at least part of the infectious agent. If infectious prions are ultimately found in the brains of transgenic mice carrying the 'GerstmannSträussler' mutation, I would consider the case for the "protein only' prion hypothesis to be convincing beyond reasonable doubt, although a rearguard action will doubtless still be fought by its opponents.
Uncomfortable as we may feel with the hypothesis, we should consider the comment made to Watson by his mentor: "when you have eliminated the impossible, whatever remains, however improbable, must be the truth . . ."22.

Charles Weissmann is in the Institut für Molekularbiologie I, University of Zürich, Hönggerberg, 8093 Zürich, Switzerland.

\title{
When galaxies get together
}

\section{Peter J. Quinn}

Is there any evolutionary connection between the two basic types of galaxy that populate the Universe - the flattened disk-like spirals (including our own Milky Way) and the rounder (triaxial) ellipticals? New work by Schweizer et al.' would appear to add fuel to the argument that most elliptical galaxies are recent arrivals, born from the aftermath of galactic collisions and mergers.

The notion that galaxies are not isolated 'islands', each containing hundreds of billions of stars, but evolve through interactions with neighbouring systems, is not new. There are several pairs of galaxies in which the partners seem to be sufficiently close for tidal forces to have distorted their otherwise symmetrical forms. It was Alar and Juri Toomre who showed $^{2}$, in 1972, that features of interacting spirals, (tails and bridges of stars and gas) are the result of the gravitational, tidal forces, not the umbilical remains from their galactic birth. The work done in distorting the galactic disks occurs at the expense of the kinetic energy of galaxies as they orbit each other. The Toomres speculated (and more detailed computer simulations ${ }^{3}$ verified) that the loss of orbital energy would lead to the complete merger of the individual disks into a single pile of stars. These star piles, Alar Toomre later proposed ${ }^{4}$, would be indistinguishable from elliptical galaxies.

Although Toomre could point to galaxies - some with a pair of identifiable but distorted disks, others showing signs of tidal distortion but only a single nucleus and yet others resembling ellipticals at the core at least - which showed signs of evolving to ellipticals, this does not show that all ellipticals evolved this way. For that notion to be persuasive, one must first prove that galactic encounters are sufficiently common to be cosmologically significant. An early estimate ${ }^{5}$ was that only one or two galaxies in every 10,000 would have merged this way, so far. But this is almost certainly an underestimate. The presence of large quantities of unseen matter in the outskirts of galaxies makes them perhaps ten times larger than their visible size and hence increases the crosssectional area they present to one another by factors of a hundred. Also, many galaxies are in bound associations, so that their relative velocities are well below the escape speed, enhancing the probability of their merging. Together, these factors raise the probability of merging for conditions seen at the present epoch towards 10 per cent.

For cosmological theories (cold dark matter theory, for example) in which large-scale structures such as clusters of galaxies are built up from a cascade of mergers of smaller objects, one must assume that large galaxies in the past were assembled from smaller components, presumably galaxies in their own right. So the past merger rate must have been larger than observations suggest for the present epoch.

So if mergers are not so rare, how many galaxies should we see merging at present? Model simulations indicate that
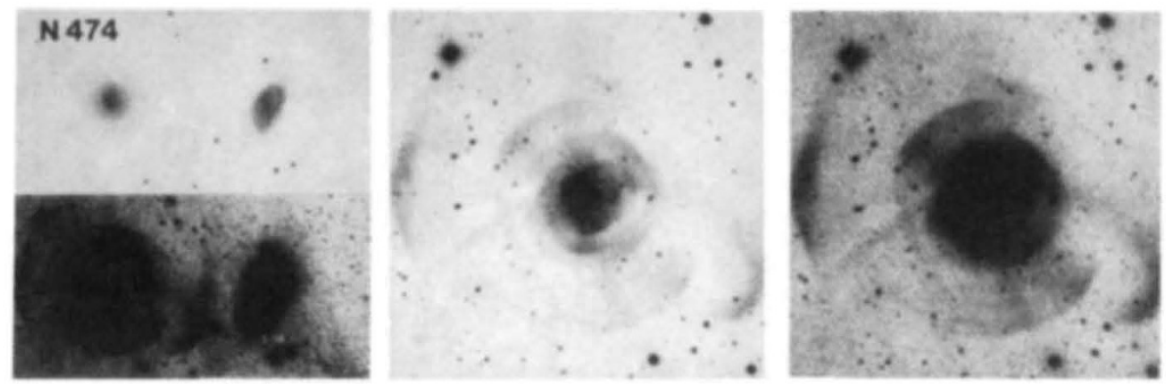

Images of the interacting lenticular galaxy NGC474 showing ripples induced by its spiral companion NGC470 (seen in left panels). Increasing degrees of structure (shells, loops and so on) are visible as fainter details are brought out by imaging techniques. (From ref. 6 .)

\section{Lattice work}

LATICE gauge theories developed to describe the symmetry-breaking transition that provided fundamental particles with masses - the Higgs mechanism - can be tested not only in high-cost, high-energy colliders but also using a beaker full of surfactant molecules in water. This suggestion, made by D.A. Huse and S. Leibler (Phys. Rev. Lett. 66, 437-440; 1991), follows from an analogy between the lattice model they use to describe the organization of the amphiphiles into 'sponge phases' and the simplest Higgs gauge theory. The analogy results from the existence of two types of sponge phase, in which the two sides of the bilayer membranes are equivalent and non-equivalent. The transition between the two phases involves spontaneous symmetry breaking, which turns out to be of the type predicted for the Higgs mechanism.

\section{Precocious puberty}

A REPORT by P.K. Manasco and colleagues in The New England Journal of Medicine $(324,227-231$; 1991) points to the biological abnormality underlying a rare sexual disorder peculiar to young males. Familial male precocious puberty is an autosomal dominant disorder marked by the onset of secondary sexual characteristics at around three years of age. The early puberty is independent of the gonadotropin hormones; but attempts to demonstrate the existence of alternative factors have not succeeded. Manasco et al. took plasma from boys unaffected and affected by the disorder and perfused it through the testicular artery of male monkeys. Plasma from affected boys produced much higher testosterone levels than did normal plasma, signifying that the disorder is caused by a circulating testis-stimulating factor. But the nature of the factor and the underlying defect remain unclear.

\section{Transparent currents}

SIGNAL-carrying currents are usually kept well separated in electronic circuits, to avoid 'crosstalk'. But J. Spector et al. now show (Appl. Phys. Lett. 58, 263-265; 1991) that the currents likely to be used in future microchips are transparent to one another, so that the wires carrying them could intersect. The trick is to use ultrapure semiconductors at ultralow temperatures, so that electrons pass directly through the wire without scattering off its constitutent ions; conventional currents, in contrast, diffuse through the wires in an unsteady shuffle. In transistors, these ballistic electrons could be manipulated by electronic 'lenses' and 'prisms'. The trend nowadays is towards incorporating optical signalling into microcircuits as light beams can intersect without crosstalk. Spector et al. suggest their finding could reverse the trend. 
it usually takes much less than 10 galactic revolutions (less than 10 per cent of the age of the Universe) for a pair of disks to merge to a single static entity following their initial close approach. Thus, there has been sufficient time for most galaxies to have undergone a merger during the history of the Universe and yet for less than 10 per cent of galaxies at any one time to show obvious signs (such as two nuclei) of recent violent interactions. When Toomre proposed his theory, there were no known long-lived indicators of mergers so that most ellipticals seemed 'clean', making it difficult to establish that all ellipticals originated through mergers.

Within the past ten years, however, improved instrumentation has revealed a wealth of detail in the cores and peripheries of ellipticals: photometrically and dynamically anomalous nuclei, rotating, for example, in the opposite sense to the whole galaxy; encircling shells of stars; disks and rings of neutral and ionized gas. These all seem to be the products of mergers, but for the most part of mergers between ellipticals and smaller companions, not necessarily between pairs of massive spiral galaxies. Models suggest that these features can persist ten times as long as it takes the original cores to fuse together.

In a previous extensive survey ${ }^{6}$, Schweizer and colleagues concluded that up to half of all bright ellipticals showed signs of a violent past (see figure). But even the others may have undergone mergers or may have been modified by acquiring material from other systems, only to have the post-interaction features erased by subsequent events. Schweizer et al. now claim ${ }^{1}$ to have found a new signature of the addition of material for many of these ellipticals. The secret is in the stellar population of the galaxies. If the added matter consists of stars younger than those already present in the elliptical or of starforming gas, the post-merger galaxy will show spectral features of young stars. Such galaxies should be apparent on a plot of spectral character versus size.

In the past, the wide spread of spectral characteristics for ellipticals has obstructed the use of this approach. But by correlating these characteristics with a selection of post-merger structures (tidal tails and so on), Schweizer et al. have now devised a quantitative index $(\Sigma)$ which measures the amount of recent merging, they claim. When plotted against the difference from the mean luminosityspectral-line-strength relationship for ellipticals, large- $\Sigma$ systems do give the most discrepant points as expected for systems with a new injection of young stars.

As the young stars age, their influence on the global spectral properties of an elliptical will fade. Hence large deviations from the mean relationships should cor- relate with recent mergers introducing young stars - except that not all the postmerger features ( $\mathrm{X}$ and box structures, for example) examined by Schweizer et al. deteriorate with time; and for those that do change, the effect on $\Sigma$ is not always in the same direction. Also, not all mergers need deliver young stars or star-forming material. And because the outcome of a merger depends sensitively on initial conditions, mergers of a similar age could give different morphologies and hence different values of $\Sigma$.

So the correlations indicated by the $\Sigma$ index may be rather too simple for something so complex as galaxy merging. It will be worth taking a closer look at the sample examined by Schweizer et al. to see why they find such a good correlation between the $\Sigma$ index and stellar content. It may well be, for example, that ellipticals with spectacular structures are those resulting from collisions involving at least one spiral galaxy. As spirals have a good supply of young stars and star-forming materials, a correlation between $\Sigma$ and stellar content could follow.

On the modelling side, there are other difficulties with the idea that all ellipticals were formed by mergers of spiral galaxies. For one thing, several of the structural features seen in ellipticals, and used by Schweizer and colleagues, are actually suppressed in collisions between partners of equal mass, and are more readily produced in collisions between an existing massive elliptical and a smaller companion. So the observed features may indicate rather a shower of smaller systems on older ellipticals. And second, purely gravitional models of galaxy mergers do not explain why there seems to be a maximum size $\left(10^{12}\right.$ solar masses $)$ for galaxies. Nor do they explain the high density of stars in elliptical galaxies. Progress will depend on observations of high-redshift galaxies? (which we see as they were in earlier epochs of the Universe; the few imaged so far show chaotic structures indicative of a violent past) to elucidate the earliest mergers and more realistic models, incorporating hydrodynamic effects and the physics of star building.

Peter J. Quinn is at Mount Stromlo and Siding Spring Observatories, The Australian National University, Private Bag, Weston Creek PO, ACT 2611, Australia.

1. Schweizer, F. et al. Astrophys. J. 364, L33-L36 (1990) 2. Toomre, A. \& Toomre, J. Astrophys. J. 178, 623-666 (1972)

Van Albada, T.S. \& Van Gorkom, J.H. Astr. Astrophys. 54 121-126 (1977).

4. Toomre, A. in The Evolution of Galaxies and Stellar Populations (eds Tinsley, B.M. \& Larson, R.B.) 401-426 (Yale University Press, 1977).

5. Tremaine, S. in The Structure and Evolution of Normal Galaxies (eds Fall, S.M. \& Lynden-Bell, D.) 67-81 (Cambridge University Press, 1981).

6. Schweizer, F. \& Seitzer, P. Astrophys. J. 328, 88-92 (1988)

7. Chambers, K.C., Miley, G.K. \& Van Breugel, W.J.M. Astro phys. J. 363, 21-39 (1990).

\section{Heavenly power}

LAST week Daedalus outlined his plan to levitate a ring of superconducting cable around the Earth. A permanent eastrunning current of a few million amps, repelled by the Earth's magnetic field, could hold the cable stably in space above the Equator. Radiation shields could keep the superconductor cold, while ionospheric drag kept it geosynchronous.

Applications would be legion. For a start, an equatorial 'space halo' would transform global communications. If positioned high enough, it could act as a passive but lossless multi-resonant loop-aerial, intercepting signals beamed up to it and reradiating them almost to the whole world. Fitted with active aerials and transponders as well, it could replace all expensive, short-lived telecommunication satellites.

Even at a mere 'auroral altitude' of 50-100 km, its Earth-horizon could be extended over most of the world by northand south-going side loops, which may be needed anyway for attitude stability. The halo could be stabilized still more firmly by connecting it to the ground with cable 'spokes'. Information, power or even equipment could then be sent up the cables. Telescopes or space-research instruments could be winched up and positioned around the halo by mobile 'halo runners'. Even a space-borne funicular railway might be possible. Lifted clear of the ground on a long cable, a funicular car could be swung across oceans and jungles by the halo runner high above, and lowered again at its destination.

A superconducting space-halo would be a powerful astronomical and geophysical instrument in its own right. As the largest possible iron-cored loop aerial, with no resistance and a lowest resonance at only a few hertz, it would open up a whole new region of high-sensitivity, low-frequency radioastronomy. Furthermore, at auroral altitude a cable carrying such a heavy steady current would powerfully perturb and attract the charged particles of the solar wind. It would be a permanent focus for auroral displays. All round the temperate zone, a glowing, shimmering, multicoloured arc would brighten the morning and evening sky, reducing street lighting bills and discouraging nocturnal crime. Better still, the space-halo would act as a lossless one-turn transformer for the a.c. components of the solar wind and the ionospheric electrojet currents. With suitable switching and controlling equipment, it could capture several gigawatts of natural, geophysical energy and transmit it down to Earth through its tethering cables. Daedalus is sending details to the US Department of Defense, which is already funding a visionary project to harness auroral currents.

David Jones 